PAPER

\title{
Short latency disconjugate vestibulo-ocular responses to transient stimuli in the audio frequency range
}

\section{P Jombík, V Bahýl}

J Neurol Neurosurg Psychiatry 2005;76:1398-1402. doi: 10.1136/jnnp.2004.047878

See end of article for authors' affiliations ......................

Correspondence to: Dr P Jombík, Department of Neurology, Laboratory of Clinical

Neurophysiology, Zvolen Hospital, Kuzmanyho nabr 28, 96089 Zvolen, Slovak Republic; bahyl@vsld. tuzvo.sk

Received 18 June 2004 In revised form 28 December 2004

Accepted 13 January 2005
Objective: To determine whether unilateral activation of the vestibular labyrinth by brief air conducted tones can elicit disconjugate reflex eye movements in healthy subjects.

Methods: 40 normal volunteers, one patient with bilateral congenital sensorineural deafness, and four patients with an acoustic neuromas were subjected to monoaural air conducted tones (125 to $6000 \mathrm{~Hz}$; $132 \mathrm{~dB}$ SPL; 3-4 ms). Eye movements were recorded by averaged EOG.

Results: The stimuli elicited bi- or triphasic transient EOG responses with a duration of about $10 \mathrm{~ms}$ and a 7-8 ms latency in 16 of 40 tested volunteers and in the patient with congenital deafness. In patients with acoustic neuromas the responses were induced only by stimuli to the healthy ear. The responses in the vertical EOG were recorded predominantly from the eye contralateral to the stimulated ear and were negligible ipsilaterally. These responses were similar to those found in patients with only one functioning labyrinth reported in a previous study. In the remaining subjects the responses were absent or barely discernible.

Conclusions: (1) Disconjugate eye movements in response to brief mechanical stimuli in this audio frequency range always indicated the side of the activated vestibular labyrinth. (2) In contrast to longer stimuli used by conventional vestibular activation methods, brief stimuli may activate only the direct monocular vestibulo-ocular pathway. This may be because the responses elicited by brief stimuli cease well before the slower indirect vestibulo-ocular subsystem can align the eyes.
$\mathrm{T}$ he primary function of the vestibulo-ocular reflex (VOR) is to provide short latency compensatory eye movements in response to head perturbations. In natural conditions the predominant frequency of these perturbations is in the rage of 1 to $10 \mathrm{~Hz}$. However, both primary canal and otolithic afferents are extraordinarily sensitive to vibrations in the frequency range of $100-500 \mathrm{~Hz}$. On the other hand, air conducted sound stimuli (ACS) mainly activate only the primary saccular afferents. ${ }^{12}$ Stimuli in the audible frequency range preferentially excite irregularly discharging vestibular neurones, and the canal afferents tend to be more irregular than the otolithic afferents. Conversely, the translational VOR (tVOR) which is mediated by the otolithic afferents more dramatically enhances its high frequency gain than the angular VOR (aVOR) which is driven by the semicircular canals. ${ }^{3}$ As both the aVOR and tVOR operate with high pass characteristics relative to head velocity input, both may respond to skull vibrations. Similarly, loud ACS might activate the sacculo-ocular reflex. ${ }^{3}$ Experimental and clinical data suggest that both vibrations and ACS can evoke vestibulo-spinal and vestibulo-ocular responses. ${ }^{4-15}$

The latency of the aVOR is approximately $10 \mathrm{~ms}$ with only slight intersubject variation, consistent with a three neurone arc. ${ }^{16-18}$ On the other hand, the mean latency of the human tVOR is approximately $30 \mathrm{~ms}$, but there is a high intersubject variability, and the latency of the tVOR can be very short in some human subjects. ${ }^{19}{ }^{20}$ Moreover, studies in animals have found that a rapid disynaptic pathway participates in mediating the tVOR. ${ }^{21-23}$ Finally, it is possible that a compensatory tVOR uses a common pathway with the aVOR.

In our previous study skull vibrations were generated by head taps or by short bone conducted sound stimuli. The later consisted of single period of $160 \mathrm{~Hz}$ logon and were delivered by a clinical bone vibrator with a repetition rate of 3 per second. We elicited transient responses with a duration of about $10 \mathrm{~ms}$ and with short latencies between 5 and $15 \mathrm{~ms}$, compatible with a disynaptic VOR. If the recorded events were generated solely by horizontal or vertical eye rotations, the amplitudes of eye movements would approach approximately $1-4^{\circ}$ in the responses elicited by head taps and $0.25-1^{\circ}$ in the bone conducted tone bursts. However, it should be taken into account that the recorded 2D signal was distorted by torsional eye movements and lid artefacts. The most surprising findings were obtained in 10 patients with unilateral vestibular loss following surgery for acoustic neuroma. They all had disconjugate responses in the vertical EOG to stimuli applied along the naso-occipital axis with enhancement of the response amplitude on the paretic side or decreased on the healthy side (fig 1B). The asymmetry ratios were statistically significant. This finding should be viewed as a consequence of vestibular loss because the incidental occurrence of the above mentioned asymmetry based on categorical data analysis was negligible. Moreover, this finding suggests that the left and right eyes of patients, in contrast with healthy subjects, should move from a different starting position through a different trajectory, or show a different eyeball-lid interaction on the healthy versus the lesioned side. However, we were uncertain whether these responses were generated by an active VOR or resulted from passive oscillatory eye movements induced by vibrations. If the later, the disconjugate eye movements would then reflect otolith mediated sustained skew torsion in patients with unilateral vestibular loss. ${ }^{15}$

The ACS can be applied monoaurally, and hence activate the vestibular labyrinth only on one side. The purpose of our current study was to compare the findings in the group of 10 patients with only one functioning labyrinth from our

Abbreviations: ACS, air conducted sound stimuli; aVOR, angular vestibulo-ocular reflex; EOG, electro-oculogram; SPL, sound pressure level; †VOR translational vestibulo-ocular reflex, ; VOR, vestibulo-ocular reflex 
A

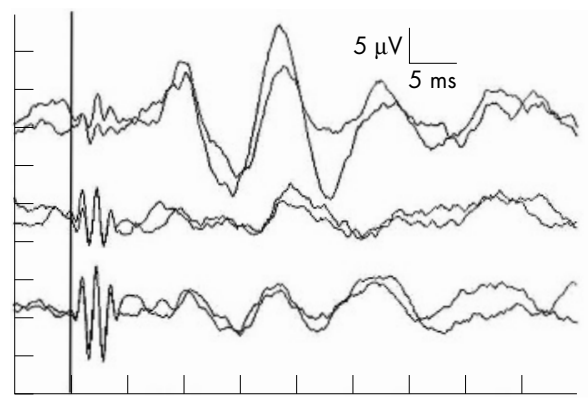

B

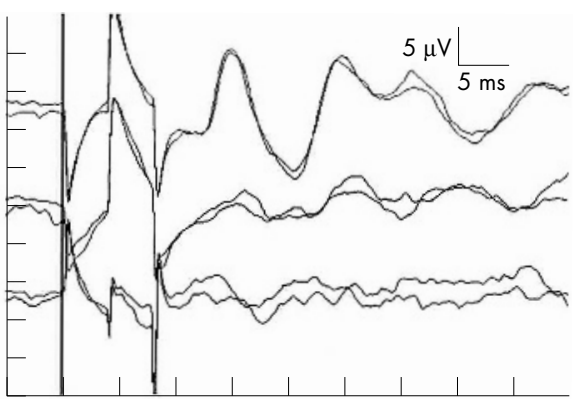

Figure 1 (A) Averaged electro-oculogram (EOG) responses to $750 \mathrm{~Hz}$ air conducted sound stimuli to right ear in a subject with bilateral congenital sensorineural deafness. (B) EOG responses to $160 \mathrm{~Hz}$ bone conducted tones applied over the inion in a subject with complete unilateral vestibular loss on the left side. Traces from top to bottom correspond to the left and right vertical and horizontal binocular channels, respectively. The vertical bold line indicates the timing of the stimuli. Following the stimulus generated by bone vibrator and headphones, an electrical artefact comprised the initial parts of the record. The signal was averaged $5 \mathrm{~ms}$ before and $45 \mathrm{~ms}$ after the stimulus and records of two averaged trials were superimposed. In both subjects the responses in the vertical EOG were disconjugate and predominated on the side opposite to the activated vestibular labyrinth; they always indicated the side of the activated labyrinth.

previous study with reflex eye movements elicited by short air conducted sound stimuli in normal subjects.

\section{METHODS \\ Subjects}

We studied 40 normal volunteers aged between 19 and 69 years (mean (SD), 42.3 (14.9)) without a history or clinical signs of vestibular or oculomotor disorder, one subject with congenital sensorineural deafness, and four patients with acoustic neuroma. Two of the latter four had undergone neuroma resection, and hearing was preserved in one of them. All control subjects, as well as the volunteer with congenital deafness, had normal responses in the head thrust test and normal vestibulo-collic responses in the sternocleidomastoid muscles. The patients with neuromas had consistent ipsilateral absence of these responses. The study was carried out in accordance with the 1964 Declaration of Helsinki protocol and with approval of the institutional ethics committee.

\section{Test procedures}

Subjects were seated upright in a dimly lit room and viewed a small illuminated target straight ahead from a $3.5 \mathrm{~m}$

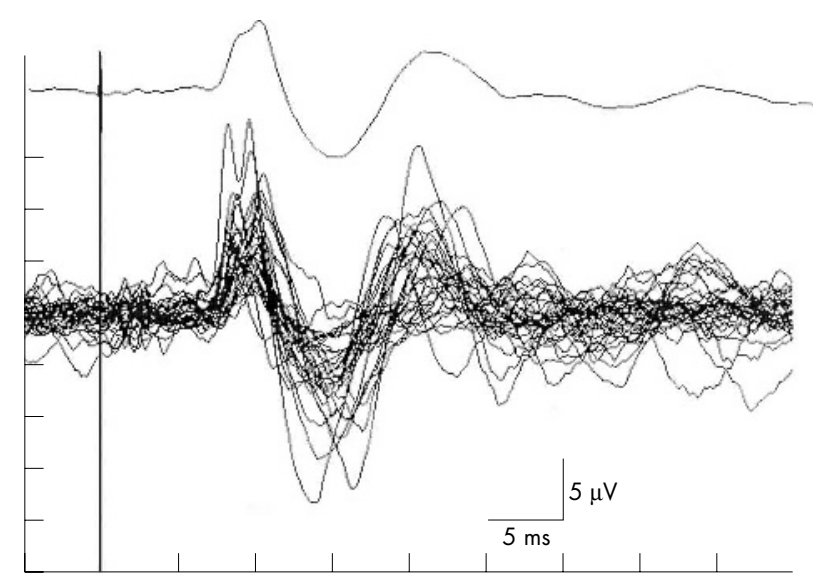

Figure 2 Averaged vertical electro-oculogram of the contralateral eye in response to monoaurally delivered air conducted sound stimuli: superimposed traces of individual averaged responses in test subjects and the grand average. Note the low intersubject variability and the machine-like precision in timing of the responses consistent with a direct vestibulo-ocular reflex response.

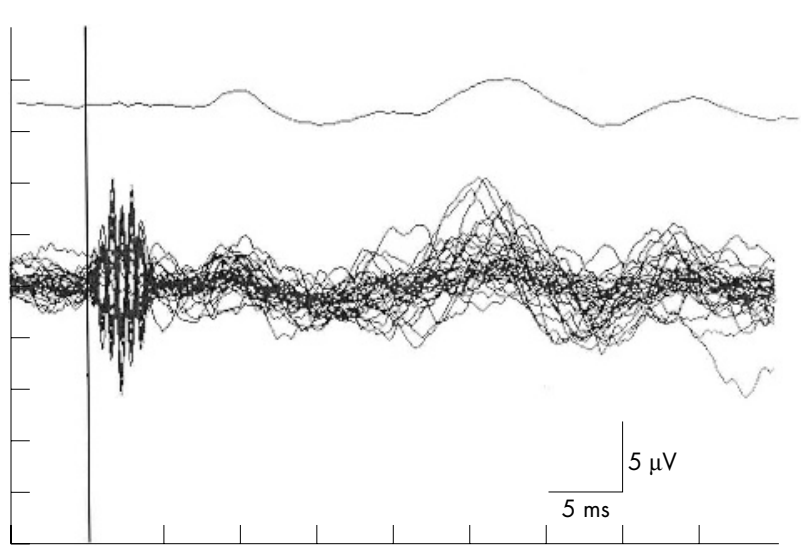

Figure 3 Averaged vertical electro-oculogram (EOG) of the ipsilateral eye in response to monoaurally delivered air conducted sound stimuli: superimposed traces of individual averaged responses in test subjects and the grand average. In contrast to the EOG responses on the opposite side, the ipsilateral EOG responses had significantly lower amplitudes with respect to the stimulated ear. However, they did not differ in any other way.

distance. Several subjects were also studied with eccentric horizontal gaze positions. Care was taken to obtain relaxation of the jaw muscles. The ACS were 125 to $6000 \mathrm{~Hz}$ logons*, spaced approximately in half octave or octave steps (see fig 4), with a duration of 3-4 ms. and intensity of $132 \mathrm{~dB}$ sound pressure level (SPL). They were delivered monoaurally through headphones with a repetition rate of 3.1 per second. Reflex thresholds were determined in four subjects with well formed responses using $10 \mathrm{~dB}$ decrements in sound intensity. Responses were recorded by means of bilateral monocular vertical, and binocular horizontal EOG. Eye movements were calibrated with $10^{\circ}$ up, down, left, and right saccades. The signal was amplified and bandpass filtered at 5-2000 Hz. Thirty responses were averaged twice, using a sampling rate of $10000 \mathrm{~Hz}$ for each channel, $5 \mathrm{~ms}$ before and $45 \mathrm{~ms}$ after each stimulus. The upward deflection of the recorded eye position signal in vertical and horizontal EOG channels corresponded to upward and rightward eye movement, respectively. With the exception of stimulation methodthat is, air conducted sound stimuli instead of head taps and

* The logon is a particular type of tone burst with a raised cosine, instead of trapezoidal enveloping function. 
bone conducted tones-the recording conditions were identical to those used in the our previous study. ${ }^{15}$

\section{Data analysis}

In addition to slow baseline drift, EOG has a rather high baseline noise level which limits resolution to $0.5-1^{\circ}$ at best. However, for a high frequency signal with a short latency that is time locked to the stimulus, an averaging technique can increase the resolution by about one order. The vertical EOG always reflects an interaction of eye and lid movements. The eyelid acts as a sliding electrode, increasingly shunting the positive corneal pole to the upper EOG electrode while covering the larger surface of the cornea. Measured potentials associated with vertical eye movements are further distorted because the upper lid does not maintain a constant position with respect to the cornea. Moreover, the negative pole of the corneoretinal dipole does not lie exactly at the fovea, but instead it is displaced nasally by about $15^{\circ}$. This means that the electrical field is not precisely aligned with the optic axis, and so torsional movements of the globe may give rise to potential changes which are misinterpreted as horizontal or vertical movements. ${ }^{24} 25$ The signal in the horizontal binocular EOG is an approximate arithmetical sum of the signals from the left and the right eye. Conjugate eye movements, and even those that are disconjugate in amplitude of movement, but not in direction of movement-that is, dissociated eye movements-cause an increase in the signal amplitude in the binocular EOG channel. Conversely, those that are disjunctive (oppositely directed) will be cancelled. Owing to the complexity of the recorded signals and the limited reliability of our recording technique, we displayed and analysed the EOG signal in microvolts and our analysis was focused on vertical eye movements. Statistical analyses were done using the Student $t$ test and common analysis of variance. Results were considered significant at $\mathrm{p}<0.05$.

\section{RESULTS}

ACS did not elicit any response, or the responses were ambiguous, in 24 subjects. In 16 subjects, stimuli in the frequency range of $750-1000 \mathrm{~Hz}$ to either ear elicited transient disconjugate biphasic or triphasic responses with duration of about $10 \mathrm{~ms}$, predominantly in the vertical EOG of the contralateral eye (fig 1A, fig 2, fig 3). Vertical EOG responses were initially directed upward and then downward. Latency of onset, defined as the first sharp decline from baseline, was about 7-8 ms, and amplitudes measured from peak to peak were in the range of $5-37 \mu \mathrm{V}$. The responses of the ipsilateral eye were in general negligible (table 1). Horizontal EOG responses were initially directed toward and then away from the stimulated ear. Above and below the range of $500-1000 \mathrm{~Hz}$ the responses tended to diminish or were absent; thus the frequency tuning was in the range already shown for vestibular activation by ear conducted sound, with thresholds between 102 and $122 \mathrm{~dB}$ SPL (fig 4). ${ }^{16}{ }^{10}$ The responses seemed to be vestibular dependent, because they were also obtained in a subject with bilateral congenital sensorineural deafness but normal vestibular function (fig 1A). However, the responses in the patients with acoustic neuroma were elicited only when the stimuli were applied to the healthy ear, but were absent with stimulation on the lesioned side. Horizontal gaze slightly increased the response amplitude on the side of the adducting eye and decreased it on the side of the abducting eye, but without a reversal of the asymmetry.

\section{DISCUSSION}

In the present study, reflex eye movements were obtained in 38 of 90 ears in response to ACS. The absence of response to stimulation of the lesioned ear in the four patients with acoustic neuroma was obvious. The stimulation intensity at $132 \mathrm{~dB}$ SPL was equivalent to $92 \mathrm{~dB}$ of normal hearing level; thus it was about the threshold for vestibular activation by ear conducted sound. ${ }^{16}{ }^{10}$ Hence, subthreshold stimulation might be one of the reasons for the absence of the responses in the remaining 48 ears.

\section{Brain stem reflexes and auditory evoked potentials}

The early Rl component of the blink reflex can generally be elicited only by stimulating the first division of the trigeminal nerve. It occurs only ipsilaterally on the side of stimulation, and possesses a latency that is somewhat longer than was

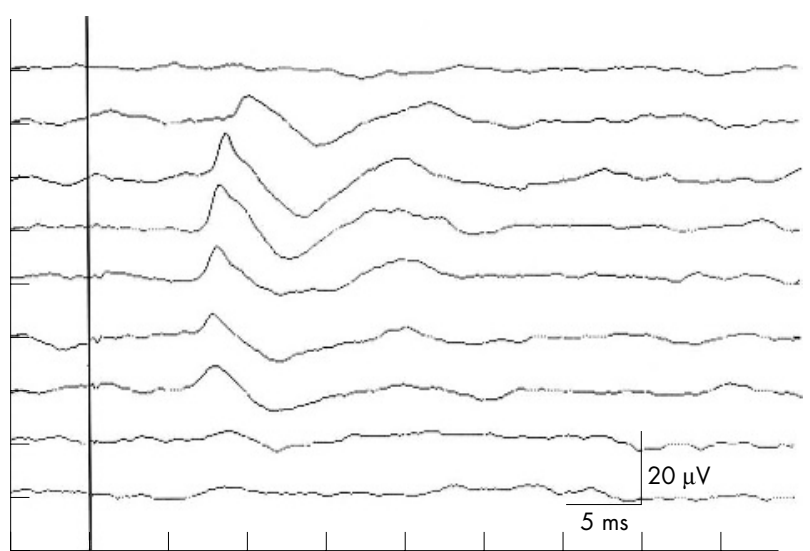

Figure 4 Frequency tuning of reflex eye movements to single periods of air conducted tones in a range from $125 \mathrm{~Hz}$ to $4 \mathrm{k} \mathrm{Hz}$ in one subject. Each trace represents averaged responses to 30 individual stimuli delivered with a repetition rate of 3.1 per second. In this case, similar to other subjects, the best frequency was at $750 \mathrm{~Hz}$. However, this subject posed an exception because of the outstandingly broad tuning curve and high sensitivity. In most subjects the responses to stimuli outside the range of 250-500 to $1000-1500 \mathrm{~Hz}$ were absent and the responses amplitudes decreased when the stimulus duration was decreased below 3 or $4 \mathrm{~ms}$.

Table 1 Contralateral and ipsilateral eye responses to stimulation of the left and right ear

\begin{tabular}{|c|c|c|c|c|c|c|c|c|c|}
\hline \multirow{3}{*}{$\begin{array}{l}\text { Stimulated } \\
\text { ear }\end{array}$} & \multicolumn{8}{|l|}{ Eye responses } & \multirow[b]{3}{*}{ AR (\%) } \\
\hline & \multicolumn{2}{|c|}{ Onset latency (ms) } & \multicolumn{2}{|c|}{ Peak 1 latency (ms) } & \multicolumn{2}{|c|}{ Peak 2 latency (ms) } & \multicolumn{2}{|c|}{ Peak 1-2 amplitude $(\mu \mathrm{V})$} & \\
\hline & Contra & Ipsi & Contra & Ipsi & Contra & Ipsi & Contra & Ipsi & \\
\hline Left & $7.20(0.33)$ & $7.49(0.71)$ & $9.57(0.70)$ & $9.85(0.81)$ & $14.67(1.33)$ & $14.51(1.26)$ & $16.08(8.68)$ & $4.61(1.81)^{* *}$ & $52.44(16.00)$ \\
\hline Right & $7.14(0.4 .6)$ & $7.57(0.61)^{*}$ & $9.70(0.87)$ & $9.83(0.79)$ & $14.59(1.01)$ & $14.81(1.40)$ & $13.83(5.62)$ & $4.11(1.52)^{* *}$ & $51.20(17.16)$ \\
\hline \multicolumn{10}{|c|}{$\begin{array}{l}\text { Values are mean (SD). } \\
\text { Contralateral and ipsilateral differences in peak } 1 \text { to peak } 2 \text { amplitudes are expressed as an asymmetry ratio }(A R) \text { calculated as } A R \%=100 \times(\text { contra-ipsi)/ } \\
\text { (contra+ipsi). } \\
\text { Statistical difference of the ipsilateral eye response to the corresponding value of the contralateral eye: }{ }^{*} p<0.05 ;{ }^{* *} p<0.01 \text {. } \\
\text { Contra, contralateral; ipsi, ipsilateral. }\end{array}$} \\
\hline
\end{tabular}


found for EOG responses in the present study. The bilateral R2 component of the blink reflex can be elicited by various sensory stimuli including loud sounds. The latency of the R2 component as well as the associated eye and lid movements generally exceeds $20 \mathrm{~ms}^{26}{ }^{27}$ Hence, any blink reflex could neither generate the short latency EOG responses nor interfere with them.

The ACS can elicit reflex responses in the ipsilateral sternocleidomastoid muscle and bilaterally in the dorsal neck muscles. However, the amplitudes of these responses are greatly dependent on the level of background muscle activity. Fairly strong tonic muscle contraction is necessary for recording the responses, even with electrodes located over the muscle belly. ${ }^{6}$ During the recording procedure these muscles were not unduly activated, so it is improbable that any significant volume conducted potential originating from them would interfere with the EOG signal. On the other hand, the sono-motor reflex of the postauricular muscle and the early components of the middle latency auditory evoked potential could be picked up by EOG with similar latency as the responses in this study. However, this myogenic potential is bilateral and the middle latency auditory evoked potential also has widespread bilateral distribution in frontal areas; thus it would probably not appear mainly in the contralateral EOG channel.

\section{Transient ocular responses versus nystagmus}

Nystagmus in response to stimuli of longer duration has slow build up. It reflects an activation of the polysynaptic pathways including the quick phase generator and the velocity storage system. The transient ocular movements in our studies were immediate responses to individual transient stimuli. We suppose that, owing to the low intensity and short duration of the stimuli as well as their separation in time (that is, more than $300 \mathrm{~ms}$ ), the velocity storage system could be not activated. However, the responses tended to disappear in the 30 to $60 \mathrm{~ms}$ following the stimulus. Though the studied subjects sometimes reported a mild degree of oscillopsia, eye movements were invisible to the naked eye.

\section{Disconjugate VOR responses}

In our previous study, disconjugate eye movements in the vertical EOG of patients with unilateral vestibular loss always indicated the side of the paretic labyrinth. ${ }^{15}$ The findings with ACS suggest that an active VOR generated the responses. Disconjugation of the eye movements might be a consequence of the spatial orientation of the unbalanced unilateral labyrinthine input.

The analysis in this report was focused on vertical eye movements, because the findings in the binocular horizontal EOG channel were hardly interpretable, for the reasons already mentioned. The latencies of the responses, about 7$8 \mathrm{~ms}$, with very low intersubject variability, were compatible with the action of a simple reflex circuit that is working with machine-like precision. This finding suggests activation of the direct disynaptic VOR pathway. The present estimate of VOR latency is consistent with recent measurements reported in other studies..$^{18}$ Nevertheless, the range of the estimated latencies was much narrower. The estimation of the VOR latency depends a great deal on the stimulus time profile. Owing to the exponential shape of the VOR response, estimation of the response onset is more difficult for stimuli of slower rise time used in other studies. The high frequency transients that were employed in the present study can elicit responses with a similar time profile and a rapid rise time, so they allow estimation of the response latency with higher precision. This was reflected in a narrower range of measured latencies in comparison with other studies.
The purpose of the VOR is to provide clear vision when the head is moving. This reflex must be cancelled by visual fixation, when the stimuli are not accompanied by head movement, or when movement of the visual target is coupled with head movement. In this study the subjects were instructed to fixate a distant target in the absence of head movement. Nevertheless, the cancellation of the tVOR and aVOR is very week up to 50 or $100 \mathrm{~ms}$ following the stimulus. ${ }^{19} 28$

If the responses were generated solely by horizontal or vertical eye rotation, the amplitude of eye movement would approach approximately $0.25-2^{\circ}$. The amplitudes were rather high, so lid artefacts could be implicated. Nevertheless, a high frequency gain enhancement might also operate. The increase in amplitude of the response in vertical EOG channel on the side of the adducting eye with horizontal gaze, and the decrease on the side of the abducting eye, could be compatible with subsequent activation of both inferior and superior oblique muscles.

Predominantly vertical transient eye movements similar to the responses that were recorded in the present study have been obtained by ACS in patients with superior canal dehiscence. ${ }^{4}$ In a study in monkeys, search coil recording of transient reflex eye movements in response to ACS revealed disconjugate horizontal movements that were similar in all four tested monkeys, but the vertical responses were highly variable and without contralateral predominance. ${ }^{5}$ We cannot say whether these discrepancies between the studies were caused by interspecies differences or by differences in experimental procedures. Nevertheless, the reproducible horizontal eye movements in monkeys suggest that the sacculo-ocular reflex was not the only one activated by the ACS. However, electrophysiological studies in cats have found only scanty polysynaptic connections from the utricle and the saccule to the vertical and oblique muscles. ${ }^{21}{ }^{29}$ These findings do not support the existence of a rapid VOR pathway from the otolithic organs to the muscles associated with vertical and torsional eye movements. Thus activation of the disynaptic pathway from the semicircular canals would be an explanation for the short latency responses in the vertical EOG that were found in our study and other, if an extrapolation of the data from cats to monkeys and humans is correct.

Eye movements generated by the VOR often have a roll component; thus the EOG has limited reliability for assessing these three dimensional eye movements. ${ }^{24} 253031$ We do not know whether conjugate eye movements about an oblique axis might not lead to an apparently disconjugate EOG pattern owing to the torsional components and different eyelid interactions on either side. However, absence of reversal of the response asymmetry with eccentric horizontal gaze positions may suggest that this asymmetry was not caused by lid artefacts, and the responses might really be disconjugate.

The basically monocular organisation of the short latency transient VOR responses is the most important issue that can arise from the findings presented here. This notion was suggested more than a century ago by Helmholz. On the other hand, Hering argued that both eyes are innervated by common command signals that yoke the eye movements (Hering's law of equal innervation), and this view gained general acceptance up to recent times. However, several studies in the last decade showed evidence that Hering's law is unlikely to be entirely correct. Studies in animals support the view that the general vertebrate bauplan is based on independent control units for each eye separately. The disconjugate nature of eye movements of monkeys during REM sleep suggests that there may be independent saccade generators for left and right eye movements. ${ }^{32}$ 
Electrophysiological studies in primates have shown that position-vestibular-pause neurones fire in relation to monocular eye position rather than to conjugate eye movements. ${ }^{33}$ Binocular coordination of saccadic eye movements and VOR depend on the state of alertness. ${ }^{32} 34$

The vestibular nuclei can be subdivided in several functional areas. Those that provide direct VOR pathways might generate separate monocular command signals and activate the twitch motor units. The indirect system, with slower execution time, might participate in the neural integration of velocity to position, using non-twitch motor units and multiply innervated muscle fibres. The indirect system might carry signal command for the alignment and stabilisation of the eyes. ${ }^{35}$

More conventional methods of vestibular activation use stimuli of rather longer duration, thus they provide enough time for the indirect VOR pathway to come into play. The audio frequency short duration vibrations that were used in our previous study and the sound stimuli presented here will elicit vestibular responses of similar short duration. These responses could depend on the more rapid direct VOR pathway that provides monocular command signals. Owing to the transient nature of the stimuli, the responses will cease well before the alignment of the eyes by the indirect pathway could begin. Thus these transient stimuli might provide a new non-invasive means for separate investigation of the direct rapid VOR pathway.

\section{Authors' affiliations}

P Jombik, Department of Neurology, Laboratory of Clinical

Neurophysiology, Zvolen Hospital, Zvolen, Slovak Republic

V Bahýl, Department of Physics and Applied Mechanics, Technical

University of Zvolen

Competing interests: none declared

\section{REFERENCES}

1 Young ED, Fernandez C, Goldberg JM. Responses of the squirrel monkey vestibular neurons to audio-frequency sound and head vibration. Acta Otolaryngol (Stockh) 1977:84:352-60.

2 Murofushi T, Curthoys IS. Physiological and anatomical study of click-sensitive primary vestibular afferents in the guinea pig. Acta Otolaryngol (Stockh) 1997; 117:66-72.

3 Paige GD, Telford L, Seidman SH, et al. Human vestibuloocular reflex and its interactions with vision and fixation distance during linear and angular head movement. J Neurophysiol 1998;80:2391-404.

4 Halmagyi GM, McGarvie LA, Aw ST, et al. The click-evoked vestibulo-ocular reflex in superior semicircular canal dehiscence. Neurology 2003;60:1172-5

5 Zhou W, Mustain W, Simpson I. Sound-evoked vestibulo-ocular reflexes (VOR) in trained monkeys. Exp Brain Res 2004;156:129-34.

6 Colebatch JG, Halmagyi GM, Skuse NF. Myogenic potentials generated by a click-evoked vestibulocollic reflex. J Neurol Neurosurg Psychiatry 1994;57:190-7.

7 Halmagyi GM, Yavor RA, Colebatch JG. Tapping the head activates the vestibular system: a new use for the clinical reflex hammer. Neurology 1995;45: 1927-9.

8 Sheykholeslami K, Murofushi T, Habibi Kermani M, et al. Bone-conducted evoked myogenic potentials from the sternocleidomastoid muscle. Acta Otolaryngol 2000;120:731-4.
9 Welgampola MS, Colebatch JG. Characteristics of tone burst-evoked myogenic potentials in the sternocleidomastoid muscles. Otol Neurotol $2001 ; 22: 796-802$

10 Welgampola MS, Rosengren SM, Halmagyi GM, et al. Vestibular activation by bone conducted sound. J Neurol Neurosurg Psychiatry 2003:74:771-8.

11 Hamann KF, Schuster EM. Vibration-induced nystagmus - a sign of unilateral vestibular deficit. ORL J Otorhinolaryngol Relat Spec 1999;61:74-9.

12 Dumas G, Michel J, Lavieille JP, et al. Semiologic value and optimum stimuli trial during the vibratory test: results of a 3D analysis of nystagmus. Ann Otolaryngol Chir Cervicofac, 2000;117:299-312.

13 Michel J, Dumas G, Lavieille JP, et al. Diagnostic value of vibration-induced nystagmus obtained by combined vibratory stimulation applied to the neck muscles and skull of 300 vertiginous patients. Rev Laryngol Otol Rhinol 2001;122:89-94.

14 Karlberg M, Aw ST, Black RA, et al. Vibration-induced ocular torsion and nystagmus after unilateral vestibular deafferentation. Brain 2003; 126:956-64.

15 Jombik P, Bahyl V. Short latency responses in the averaged electro-oculogram elicited by vibrational impulse stimuli applied to the skull: can they reflect the vestibulo-ocular reflex function? J Neurol Neurosurg Psychiatry 2005;76:222-8.

16 Maas EF, Heubner WP, Seidman SH, et al. Behavior of the human horizontal vestibulo-ocular reflex in response to high-acceleration stimuli. Brain Res 1989:499:153-6.

17 Aw ST, Halswanter T, Halmagyi GM, et al. Three-dimensional vector analysis of the normal human vestibuloocular reflex in response to high-acceleration head rotations. I. Responses in normal subjects. J Neurophysiol 1996;76:4009-20

18 Collewijn H, Smeets JBJ. Early components of the human vestibulo-ocular response to head rotation: latency and gain. J Neurophysiol 2000;84:376-89.

19 Gianna CC, Gresty MA, Bronstein AM. The human linear vestibulo-ocular reflex to transient accelerations: visual modulation of suppression and enhancement. J Vestibular Res 2000;10:227-38.

20 Crane BT, Tian J, Wiest G, et al. Initiation of the human heave linear vestibuloocular reflex. Exp Brain Res 2003;148:247-55.

21 Uchino $Y$, Sasaki M, Sato $H$, et al. Utriculoocular reflex arc of the cat. J Neurophysiol 1996;76:1896-903.

22 Bush GA, Miles FA. Short-latency compensatory eye movements associated with a brief period of free fall. Exp Brain Res 1996;108:337-40.

23 Angelaki DE, McHenry MQ. Short-latency primate vestibuloocular responses during translation. J Neurophysiol 1999:82:1651-4.

24 Collewijn H, Van Der Steen J, Steinman RM. Human eye movements associated with blinks and prolonged eyelid closure. J Neurophysiol 1985;54:11-27.

25 Lueck CJ, Kennard C. Oculography and techniques for eye movement recording. In: Daroff R, Neetens A, eds. Neurological organization of ocular movement. Berkeley, Ca: Kugler and Ghedini, 1990:485-502.

26 Rushworth G. Observation on blink reflexes. J Neurol Neurosurg Psychiatry 1962;25:93-108

27 Bour L, Aramideh M, Ongerboer de Visser BW. Neurophysiological aspects of eye and eyelid movements during blinking in humans. J Neurophysiol 2000;83:166-76.

28 Crane BT, Demer JL. Latency of voluntary cancellation of the human vestibuloocular reflex during transient yaw rotation. Exp Brain Res 1999;127:67-74.

29 Isu N, Graf W, Sato H, et al. Sacculo-ocular connectivity in cats. Exp Brain Res 2000;131:262-8.

30 Thurtell MJ, Kurin M, Raphan T. Role of muscle pulleys in producing eye position- dependence in the angular vestibuloocular reflex: a model-based study. J Neurophysiol 2000;84:639-50.

31 Raphan T, Cohen B. The vestibulo-ocular reflex in three dimensions. Exp Brain Res 2002;145:1-27.

32 Zhou W, King WM. Binocular eye movements not coordinated during REM sleep. Exp Brain Res 1997;117:153-60

33 McConville K, Tomlinson RD, King WM, et al. Eye position signals in the vestibular nuclei: consequences for models of integrator function. J Vestib Res 1994:4:391-400

34 Fluur $\mathrm{E}$, Mellstrom A. The otolith organs and their influence on oculomotor movements. Exp Neurology 1971;30:139-47.

35 Buttner-Ennever JA, Horn AKE. The neuroanatomical basis of oculomotor disorders: the dual motor control of extraocular muscles and its possible role in proprioception. Curr Opin Neurol 2002;15:35-43. 